\title{
Distinct Alteration Patterns of Resting-State Functional Connectivity of the Corticostriatal Circuits Effected by Cigarette Smoking in Mild Cognitive Impairment Patients and Cognitively Normal subjects
}

\section{Tiantian Qiu}

Linyi People's Hospital https://orcid.org/0000-0002-9428-4117

\section{Fei Xie}

\section{Linyi People's Hospital}

\section{Qingze Zeng}

Zhejiang University School of Medicine Second Affiliated Hospital

\section{Zhujing Shen}

Zhejiang University School of Medicine Second Affiliated Hospital guijin Du

Linyi People's Hospital

\section{Xiaopei Xu}

Zhejiang University School of Medicine Second Affiliated Hospital

\section{Chao Wang}

Zhejiang University School of Medicine Second Affiliated Hospital

Xiaodong Li

Linyi People's Hospital

Xiao Luo

Zhejiang University School of Medicine Second Affiliated Hospital

\section{Kaicheng Li}

Zhejiang University School of Medicine Second Affiliated Hospital

\section{Peiyu Huang}

Zhejiang University School of Medicine Second Affiliated Hospital

\section{Tianyi Zhang}

Tongde Hospital Of Zhejiang Province

Jinling Zhang

Linyi People's Hospital

\section{Shouping Dai}




\section{Minming Zhang ( $\nabla$ zhangminming@zju.edu.cn )}

Zhejiang University School of Medicine Second Affiliated Hospital

\section{Research Article}

Keywords: Mild cognitive impairment, cigarette smoking, striatum, corticostriatal circuits, resting-state functional connectivity.

Posted Date: October 18th, 2021

DOl: https://doi.org/10.21203/rs.3.rs-959685/v1

License: (c) (1) This work is licensed under a Creative Commons Attribution 4.0 International License. Read Full License 


\section{Distinct Alteration Patterns of Resting-State Functional Connectivity of the Corticostriatal Circuits Effected by Cigarette Smoking in Mild Cognitive Impairment Patients and Cognitively Normal subjects}

Tiantian Qiu $^{\mathrm{a},{ }^{\dagger}}$, Fei Xie ${ }^{\mathrm{b},{ }^{\dagger}}$, Qingze Zeng ${ }^{\mathrm{c},}{ }^{\dagger}$, Zhujing Shen ${ }^{\mathrm{c}}$, Guijin, Du ${ }^{\mathrm{a}}$, Xiaopei $\mathrm{Xu}^{\mathrm{c}}$, Chao Wang ${ }^{\mathrm{c}}$, Xiaodong $\mathrm{Li}^{\mathrm{a}}$, Xiao Luo ${ }^{\mathrm{c}}$, Kaicheng Lic ${ }^{\mathrm{c}}$, Peiyu Huang ${ }^{\mathrm{c}}$, Tianyi Zhang ${ }^{\mathrm{d}}$, Jinling Zhang ${ }^{\mathrm{e}, *}$, Shouping Dai $^{\mathrm{a}, *}$, Minming Zhang ${ }^{\mathrm{c}, *}$, for the Alzheimer's Disease Neuroimaging

aDepartment of Radiology, Linyi People's Hospital, Linyi, China

${ }^{b}$ Department of Equipment and medical engineering, Linyi People's Hospital, Linyi, China

cDepartment of Radiology, The Second Affiliated Hospital of Zhejiang University School of Medicine, Hangzhou, China

${ }^{\mathrm{d}}$ Department of Neurology, Tongde Hospital of Zhejiang Province, Hangzhou, China

${ }^{\text {e}}$ Cancer Center, Linyi People's Hospital, Linyi, China

${ }^{\dagger}$ These authors have contributed equally to this work and share first authorship.

*These authors have contributed equally to this work and share senior authorship.

A running title: Cigarette smoking effects on MCI

Abstract: 245 words

Manuscript :2991 words

Correspondence author:

Minming Zhang, zhangminming@,zju.edu.cn

Tel./Fax: +86057187315255

Department of Radiology, The Second Affiliated Hospital of Zhejiang University School of Medicine, Hangzhou, China

Address: No.88 Jiefang Road, Shang-cheng District, Hangzhou 310009, China.

Shouping, Dai, daishouping@sina.cn 
Tel./Fax: +86 05398225769

Department of Radiology, Linyi People's Hospital, Linyi, China

Address: No.27 east section of Jiefang Road, Lanshan District, Linyi 276000, China

Jinling Zhang, jinlingzhang931@,163.com

Tel./Fax: +86 05398097161

Cancer Center, Linyi People's Hospital, Linyi, China

Address: No.27 east section of Jiefang Road, Lanshan District, Linyi 276000, China 


\begin{abstract}
To explore the interaction effects of smoking status (non-smoking vs. smoking) and disease (cognitively normal (CN) vs. MCI) based on resting-state functional connectivity (rsFC) of the corticostriatal circuits. We included $304 \mathrm{CN}$ non-smokers, $44 \mathrm{CN}$ smokers, $130 \mathrm{MCI}$ non-smokers, and 33 MCI smokers. The seed-based rsFC of striatal subregions (caudate, putamen, and nucleus accumbens [NAc]) with the whole-brain voxel was calculated. Furthermore, we performed mixed effect analysis to explore the interaction effects between smoking status and disease. Significant interaction effects were detected between: (1) right caudate and left inferior parietal lobule (IPL); (2) right putamen and bilateral cuneus; (3) bilateral NAc and bilateral anterior cingulate cortex (ACC). The post-hoc analyses revealed that the $\mathrm{CN}$ smokers showed increased rsFC between right caudate and left IPL compared to non-smokers; while the MCI smokers demonstrated decreased rsFC between right putamen and cuneus, and increased rsFC between bilateral NAc and ACC compared to non-smokers. In MCI smokers, the rsFC value between left NAc and ACC was positively correlated with Semantic Verbal Fluency $(\mathrm{SVF}, \mathrm{r}=0.387, p=0.026)$, and the rsFC value between right NAc and ACC was positively correlated with SVF $(\mathrm{r}=0.390, p=0.025)$, Wechsler memory scale-logical memory (WMS-LM) immediate recall $(\mathrm{r}=0.378, p=0.03)$, and WMS-LM delayed recall $(\mathrm{r}=0.367, p=0.036)$. Our findings suggest that chronic nicotine exposure may lead to functional connectivity alterations of corticostriatal circuits in MCI patients, and the pattern is different from $\mathrm{CN}$ smokers.
\end{abstract}

Keywords: Mild cognitive impairment; cigarette smoking; striatum; corticostriatal circuits; resting-state functional connectivity. 


\section{Introduction}

Alzheimer's disease (AD) is the most common type of dementia in elderly, which is characterized by progressive and irreversible cognitive decline. Mild cognitive impairment (MCI) is the prodromal phase of $\mathrm{AD}$ and is associated with a high risk of progression to $\mathrm{AD}$ (Larrieu et al., 2002). Recent clinical trials of AD targeting the amyloid hypothesis have failed (Knopman, 2019), which led us to think that controlling risk factors could curb the progression of AD. Cigarette smoking is recognized as one of the important risk factors of AD (Rusanen et al., 2011). Therefore, exploring the effects of smoking on brain functional activity in MCI patients is of great significance for guiding early clinical intervention.

Nicotine is the psychoactive component of cigarette smoking which causes the addiction process. It excites dopaminergic neurons of the mesencephalon by activating the nicotinic acetylcholine receptors(nAChRs) on the dopaminergic somata (Livingstone and Wonnacott, 2009), inducing the dopamine release in the target areas, particularly the striatum(Pontieri et al., 1996; Balfour, 2004; Subramaniyan and Dani, 2015). Striatum dopamine system dysfunction, such as lower dopamine receptors availability (Okita et al., 2016) and reduced [11C]raclopride binding potential (an indirect measure of dopamine release) by positron emission tomography (Brody et al., 2004) has been reported in nicotine addiction. Specifically, the striatum is comprised of the dorsal striatum (DS) and ventral striatum (VS). The DS (mainly the caudate and putamen) is involved in motor control and cognitive functions; while VS (mainly the nucleus accumbens [NAc]), the ventral extension of the DS, plays a central role in reward, development of addictive behaviors and habit formation (Haber, 2016). Furthermore, the DS and VS showed wide connections with cortical and limbic regions, such as the prefrontal cortex (PFC), orbital frontal cortex, anterior cingulate cortex (ACC), insula, and hippocampus, which constitutes the close corticostriatal circuits that contribute to the 
processes of addiction and cognition(Looi and Walterfang, 2013; Steiner and Van Waes, 2013).

The abnormal functional connectivity (FC) of the corticostriatal circuits has been observed in healthy smokers and $\mathrm{AD} / \mathrm{MCI}$ patients. For example, the $\mathrm{FC}$ of the striatum with dorsal ACC was negatively correlated with the severity of nicotine addiction in healthy smokers(Hong et al., 2009).

In addition, $\mathrm{AD}$ and $\mathrm{MCI}$ patients exhibited abnormal connectivity between the striatum and cortical regions, including the PFC, medial frontal cortex, and middle temporal cortex(Tam et al., 2015; Quan et al., 2020; Wang et al., 2020). Another study also found that reduced FC of striatum with precuneus was correlated with memory decline in patients with AD (Anderkova et al., 2017). However, the exact effects of smoking on FC alterations of corticostriatal circuits in MCI patients remains unclear.

This study aimed to investigate the smoking effects on the FC alterations of the corticostriatal circuits in patients with MCI. Both DS and VS subregions (caudate, putamen, and NAc) were chosen as the seeds for FC analyses. Based on the converging target of corticostriatal circuits in both cigarette smoking and $\mathrm{AD}$, we hypothesized that there is an interaction effect between smoking status and disease.

\section{Methods}

\section{Study participants}

The data in this study was obtained from the Alzheimer's disease Neuroimaging Initiative (ADNI) database (http://adni.loni.usc.edu/). The Institutional Review Board approved the study of all the participating institutions. Informed written consent was obtained from all participants at each site. Based on the ADNI 3 databases, we identified 378 right-handed cognitively normal subjects and 182 MCI patients with complete neuropsychological evaluations, structural magnetic resonance imaging (MRI), and resting-state functional MRI (rsfMRI) scans. We downloaded data from the 
ADNI database before March 2021. The criteria for MCI and CN have been described in detail in previous studies(Qiu et al., 2016). Then, we classified MCI and CN subjects into non-smoking and smoking subgroups based on self-report smoking history. Non-smoking was defined as participants who reported they never smoked cigarettes during their lifetime, and smoking was defined as the presence of any history of smoking. After screening, 49 participants (including $29 \mathrm{CN}$ non-smokers, $1 \mathrm{CN}$ smoker, and $19 \mathrm{MCI}$ non-smokers) were excluded for excessive head motion (details later). Finally, $304 \mathrm{CN}$ non-smokers, $44 \mathrm{CN}$ smokers, $130 \mathrm{MCI}$ non-smokers, and 33 MCI smokers entered subsequent analyses.

\section{Demographic and clinical characteristics}

Demographic factors including age, sex, and education level, and vascular risk factors including hypertension, diabetes mellitus, and hypercholesterolemia were assessed. Hypertension was defined as systolic blood pressure $>140 \mathrm{~mm} \mathrm{Hg}$, diastolic blood pressure $>90 \mathrm{~mm} \mathrm{Hg}$, medical history of hypertension, or with antihypertensive medication treatment. Diabetes mellitus was defined as random blood glucose $\geq 11 \mathrm{mmol} / \mathrm{dL}$, medical history of diabetes mellitus, or treatment with glucose-lowering medication. Hypercholesterolemia was defined as random blood cholesterol $\geq 11 \mathrm{mmol} / \mathrm{L}$, medical history of hypercholesterolemia, or treatment with lipid-lowering medication. Neuropsychological tests in different cognitive domains, including memory (Wechsler memory scale-logical memory, WMS-LM, immediate and delayed recall); attention (Trail-Making Test, Part

A, TMT-A); execution (Trail-Making Test, Part B, TMT-B); and language (Semantic Verbal Fluency, SVF) were included.

\section{Imaging data acquisition}

All subjects were scanned using a 3.0-Tesla Philips MRI scanner. The structural MRI images were acquired using a 3D MPRAGE T1-weighted sequence with the following parameters: repetition 
time $(\mathrm{TR})=2300 \mathrm{~ms}$; echo time $(\mathrm{TE})=2.98 \mathrm{~ms}$; inversion time $(\mathrm{TI})=900 \mathrm{~ms} ; 170$ sagittal slices; within plane FOV $=256 \times 240 \mathrm{~mm}^{2}$; voxel size $=1 \times 1 \times 1 \mathrm{~mm}^{3}$; flip angle $=9^{\circ} ;$ bandwidth $=240$ Hz/pix. The rsfMRI images were obtained using an echo-planar imaging sequence with the following parameters: 197 time points; $\mathrm{TR}=3000 \mathrm{~ms}$; $\mathrm{TE}=30 \mathrm{~ms}$; slice thickness $=3.39 \mathrm{~mm}$; spatial resolution $=3.39 \times 3.39 \times 3.39 \mathrm{~mm}^{3}$; flip angle $=90^{\circ}$; and matrix $=64 \times 64$.

\section{Preprocessing of rsfMRI and structural MRI data}

We preprocessed rsfMRI data using the Data Processing \& Analysis for Brain Imaging (DPABI, http://rfmri.org/dpabi) (Yan et al., 2016) with Statistical Parametric Mapping software (SPM12, http://www.fil.ion.ucl.ac.uk/spm/) on the MATLAB platform (MathWorks, Natick, MA, USA). The first ten time points of rsfMRI data were discarded due to the instability of the initial MRI signal and the subjects' adaptation to the scanning noise. The remaining 187 images were corrected for timing differences between each slice and head motion (6-parameter rigid body). Subjects with more than $3.0 \mathrm{~mm}$ maximum displacement in any of the $\mathrm{x}, \mathrm{y}$, or $\mathrm{z}$ directions or $3.0^{\circ}$ of any angular motion were discarded (including $29 \mathrm{CN}$ non-smokers, $1 \mathrm{CN}$ smoker, and $19 \mathrm{MCI}$ non-smokers). Subsequently, rsfMRI images were spatially normalized to the standard EPI template and resampled into $3 \times 3 \times 3 \mathrm{~mm}^{3}$. The rsfMRI images were spatially smoothed with a Gaussian kernel of $6 \times 6 \times 6 \mathrm{~mm}^{3}$ full width at half maximum. Finally, linear trends and temporally filter $(0.01 \mathrm{~Hz}<$ $\mathrm{f}<0.08 \mathrm{~Hz}$ ) were performed. Nuisance covariate regression was performed to minimize physiological noise using the Friston-24 head motion parameters (six head motion parameters, six head motion parameters from the previous time point, and the 12 corresponding squared items), as well as white matter (WM) signal and corticospinal fluid (CSF) signal. In addition, the framewise displacement (FD) jenkinson value of each subject was calculated to correct for the head motion artifacts. 
The T1-weighted images were preprocessed and analyzed using the Computational Anatomy Toolbox (CAT12, http://dbm.neuro.uni-jena.de/cat/) and SPM12. The images were bias-corrected, tissue-classified (grey matter (GM), WM, and CSF), and registered using linear (12 parameter affine) and non-linear transformations (warping) within the CAT12 default preprocessing pipeline.

\section{Striatum-based resting-state functional connectivity (rsFC) analysis}

In order to explore the interaction effects between smoking status and disease on striatum-based rsFC, both left and right striatal subregions (i.e., caudate, putamen, and NAc) were chosen as the seeds for rsFC analyses according to Harvard-Oxford subcortical structural atlas. Dynamic brain connectome (DynamicBC) analysis toolbox (http://restfmri.net/forum/DynamicBC)(Liao et al., 2014) was used to create individual subject seed-to-voxel connectivity maps. First, each mask was resampled to the dimension of our normalized functional image with $3 \times 3 \times 3$ voxel size for seedbased rsFC analyses. Next, the rsFC maps were generated by calculating the Pearson correlation between the time course of striatal subregions and whole brain areas. Finally, the resulting rsFC maps were transformed to $\mathrm{Z}$ maps using Fisher's $\mathrm{Z}$ transformation.

\section{Propensity score matching}

Propensity score matching (PSM) implanted in SPSS version 26 was performed to balance the differences in demographic features between non-smoking and smoking subgroups in CN and MCI, and to reduce the bias due to confounding factors. A 1: 2 matching was used to pair subjects with $\mathrm{CN}$ smokers and MCI smokers based on the following covariates: age, sex, and education level. After PSM, 86 CN non-smokers, 44 CN smokers, 62 MCI non-smokers, and $32 \mathrm{MCI}$ smokers were selected from the initial population. The demographic and clinical data were summarized in Supplementary Table 1. The interaction regions on rsFC of the striatal subregions were displayed in Supplementary Fig 1 and Table 2, and the results are consistent with those before PSM. 


\section{Statistical analysis}

The statistical analyses of demographics and neuropsychological data were performed using SPSS 26.0 statistical software. We performed a group-level analysis using one-way analysis of variance (ANOVA) for continuous variables. If group-level test results were significant, post-hoc pairwise comparisons were performed (Bonferroni multiple comparison correction in parametric tests, Dunn multiple comparison tests in nonparametric tests). Binary data, such as sex, hypertension, diabetes mellitus, and hypercholesterolemia were compared between groups using a chi-square test.

The statistical analyses of the rsFC of striatal subregions(left and right caudate, putamen, and NAc) were performed using the DPABI toolbox(Yan et al., 2016). Specifically, we performed a 2 $\times 2$ mixed effect analysis to explore the interaction effects of smoking status (non-smoking vs. smoking) and disease (CN vs. MCI). Age, sex, education level, head motion (FD value) and vascular risk factors were used as covariates. To control the effect of cortical atrophy on the functional analysis, normalized modulated (with the volumetric information encoded) GM maps were used as covariate images. The threshold was set to the voxel level at $p<0.005$ and the cluster level at $p<0.05$ after Gaussian random field (GRF) correction. To further understand how smoking status and disease interacted on the rsFC of the striatal subregions, we extracted the mean rsFC values from the interaction regions and further performed post-hoc pairwise comparisons $(p<0.05$, Bonferroni correction). At last, partial correlation analysis was performed to investigate the correlation between the mean rsFC values of interaction regions and neuropsychological scores with age, sex, and education level as covariates $(p<0.05)$.

\section{Results}

\section{Demographic and clinical characteristics}

The demographic and clinical characteristics of the four subgroups were summarized in Table $\mathbf{1}$. 
The MCI smokers $(76.61 \pm 7.54$ years $)$ were older $(p<0.05)$ than MCI non-smokers $(72.98 \pm 7.25$ years). The $\mathrm{CN}$ smokers had a predominance of females than other subgroups $(p=0.001)$. There were no significant differences between subgroups in vascular risk factors $(p>0.05)$. The MCI patients (smokers and non-smokers) showed significantly lower neuropsychological performance on memory, attention, execution, and language than $\mathrm{CN}$ subjects $(p<0.05)$. The smokers $(\mathrm{CN}$ and MCI) showed no significant differences in neuropsychological performance compared to nonsmokers $(p>0.05)$.

\section{Smoking status $\times$ disease interaction on $\mathrm{rsFC}$ of the striatal subregions}

The significant interaction effects of smoking status (non-smoking vs. smoking) $\times$ disease $(\mathrm{CN}$ vs $\mathrm{MCI}$ ) on rsFC of the striatal subregions were detected between: (1) right caudate and left inferior parietal lobule (IPL) (Fig. 1A); (2) right putamen and bilateral cuneus (Fig. 1B); (3) left NAc and bilateral anterior cingulate cortex (ACC) (Fig. 1C); and (4) right NAc and bilateral ACC (Fig. 1D). The interaction effects results of rsFC analyses were summarized in Table 2.

Then, post-hoc region-of-interest analyses were performed for the brain regions showing interaction effects (Fig. 2). Especially, the $\mathrm{CN}$ smokers showed increased rsFC of right caudate to left IPL than other subgroups. In MCI smokers, decreased rsFC of right putamen to cuneus and increased rsFC of bilateral NAc to ACC were observed when compared to MCI non-smokers.

\section{Correlation between rsFC of the striatal subregions and cognition}

We studied the relationships between rsFC values of the interaction regions (left IPL, bilateral cuneus, and bilateral ACC) and different cognition domains (Table 3). In MCI smokers, we found that the rsFC value between left NAc and ACC was positively correlated with language (SVF, $\mathrm{r}=$ 0.387, $p=0.026$ ); the rsFC value between right NAc and ACC was positively associated with language $(\mathrm{SVF}, \mathrm{r}=0.390, p=0.025)$ and memory (WMS-LM immediate recall, $\mathrm{r}=0.378, p=0.03$; 
delayed recall, $\mathrm{r}=0.367, p=0.036)($ Fig. 3). After Bonferroni correction $(p<0.05 / 20)$ for multiple comparisons, there was no significant correlation between rsFC values of the interaction regions and neuropsychological scores.

\section{Discussion}

Our study explored the interaction effects between cigarette smoking status and disease based on the rsFC of the striatal subregions. In $\mathrm{CN}$ subjects, the smokers showed increased rsFC between the caudate and IPL compared to non-smokers. In MCI patients, the smokers demonstrated decreased rsFC between the putamen and cuneus, and increased rsFC between NAc and ACC compared to non-smokers. Our findings suggested that the effects of cigarette smoking exerted on corticostriatal circuits connectivity are different in CN smokers and MCI smokers.

The CN smokers showed increased rsFC between the caudate and IPL compared to non-smokers. There is increasing evidence that habitual mechanism plays a vital role in addiction(Yalachkov et al., 2009; Jasinska et al., 2014). Individuals with greater nicotine dependence severity had increased engagement of motor preparation circuits, suggesting increased reliance on habitual behavior(Claus et al., 2013). Specifically, the IPL is the key brain region controlling the conscious motor intention 'wanting to move', which specifies a general goal to be reached before movement planning(Desmurget and Sirigu, 2012). Previous studies demonstrated that smoking cue-induced activation in the IPL, which is associated with nicotine dependence severity(Engelmann et al., 2012; Yalachkov et al., 2013). Therefore, increased connectivity between the caudate and IPL indicated that smokers could have intense motor intention on craving for smoking before act to smoke. Furthermore, we observed decreased rsFC between the caudate and IPL in MCI smokers when compared with $\mathrm{CN}$ smokers. Besides the function of motor intention, the IPL is also a functional core of the default mode network (DMN), which plays a crucial role in episodic memory 
retrieval(Buckner et al., 2008) and is vulnerable to AD neuropathology(Palmqvist et al., 2017; Chhatwal et al., 2018). Disrupted FC of the IPL/DMN has been widely reported in MCI and AD patients(Dennis and Thompson, 2014; Zhou and Seeley, 2014; Ibrahim et al., 2021). Moreover, a progressive declining trend of structural and functional connections within the DMN has also been observed from NC to MCI and then to AD patients(Zhu et al., 2013). Taken together, the IPL could be a key interaction region associated with smoking-related motor intention and cognition decline.

In MCI smokers, we found decreased rsFC of the putamen to cuneus when compared to nonsmokers. The cuneus is a part of the visual associated cortex and participates in visual selective attention by relaying top-down information from the attention network to the visual cortex(Ninomiya et al., 2012). In addiction-related studies, chronic tobacco smoking could cause a decline in attention(Conti et al., 2019; Nadar et al., 2021). Moreover, chronic smokers also showed reduced gray matter volume and density in visual attention-related regions, such as the cuneus, lingual gyrus, and other occipital cortices(Gallinat et al., 2006). In patients with MCI, cognitive decline involve in episodic memory, visuospatial skills, and attention have been frequently reported(Nelson and O'Connor, 2008). Importantly, some declines in episodic memory might stem from early deteriorations in visual attention which might influence later memory(McDonough et al., 2019). In some rsfMRI studies, aberrant spontaneous low-frequency brain activity and regional homogeneity in the visual network have been observed in MCI patients(Pan et al., 2017; Zhen et al., 2018). Additionally, based on large-scale brain networks analysis, MCI patients also showed abnormal FC in the attention network(Wang et al., 2015; Sullivan et al., 2019).

It is worth noting that the MCI smokers also showed increased rsFC between the NAc and ACC compared to non-smokers. Increased intrinsic brain activity in MCI smokers had been reported in previous work(Zhang et al., 2020), which supports our finding. The ACC shows specific 
interconnections with other PFC and dopaminergic striatal subregions, particularly the NAc, constituting the important frontostriatal reward circuit, which plays a key role in nicotine addiction(Haber and Knutson, 2010). As the primary ingredient of tobacco, nicotine shows a high affinity to nAChRs, which are widely distributed in the functional nodes of frontostriatal circuit(Hampel et al., 2018). Importantly, the nicotine could stimulate nAChRs, especially the $\alpha 4 \beta 2$ and $\alpha 7$ subtypes, which protected neurons from $A \beta$-induced toxicity in vitro(Mehta et al., 2012). Therefore, the increased $\mathrm{rsFC}$ within the frontostriatal reward circuit in MCI smokers might indicate the compensatory mechanism of smoking against disrupted brain function caused by AD pathology to some extent. Furthermore, correlation analysis revealed that increased rsFC between NAc and ACC was positively correlated with memory and language in MCI smokers, which further emphasized the role of frontostriatal reward circuit in integrating information across the addiction and cognition.

The current study has several limitations. First, despite the large sample size of the ADNI database, the smoking subgroups are relatively small due to a positive selection from the population in respect to health and lifestyle in ADNI. Future studies with a larger sample size are needed to verify our work. Second, smoking history in the ADNI database is defined by subjective self-report from the medical record, including former and current smokers. Most participants lack detailed records such as the number, duration, and status (former or current). Future studies may further explore the effects of different smoking degrees or statuses on cognition. Finally, this crosssectional study lacks clinical follow-up to make any possible inference between smoking and AD. Thus, longitudinal studies are needed to determine whether the FC alterations of corticostriatal circuits in smokers are related to disease progression.

\section{Conclusions}


In conclusion, chronic nicotine exposure may lead to functional connectivity disruption between striatum, cuneus and ACC in patients with MCI. Moreover, the effects of cigarette smoking exerted on corticostriatal circuits connectivity are different in $\mathrm{CN}$ smokers and MCI smokers.

\section{Acknowledgements}

None.

\section{Ethical approval}

All procedures performed in studies involving human participants were in accordance with the ethical standards of the institutional and/or national research committee and with the 1964 Helsinki declaration and its later amendments or comparable ethical standards.

\section{Consent to Participate}

Written informed consent was obtained from all participants and/or authorized representatives and the study partners before any protocol-specific procedures were carried out in the ADNI study.

\section{Consent to Publish}

All authors agree to publish this article in Brain Imaging and Behavior.

\section{Authors Contributions}

Author contributions included conception and study design (TTQ, FX, JLZ, SPD, and MMZ), data collection or acquisition (TTQ, QZZ, ZJS, and GJD), statistical analysis (TTQ), interpretation of results (TTQ, FX, and XPX), drafting the manuscript work or revising it critically for important intellectual content (CW, XDL, XL, KCL, PYH, and TYZ) and approval of final version to be published and agreement to be accountable for the integrity and accuracy of all aspects of the work (All authors).

\section{Fundings}

This study was funded by the National Key Research and Development Program of China (Grant 
No. 2016YFC1306600), Linyi Science and Technology Development Program (Grant No. 202020024), Shandong Medicine and Health Science and Technology Program (Grant No. 202009010844), Zhejiang Provincial Natural Science Foundation of China (Grant No. LY21H180003), and National Natural Science Foundation of China (Grant No. 81901707, 82001766 and 81901721$)$. The data collection and sharing for this project were funded by the ADNI (National Institutes of Health Grant U01 AG024904) and DOD ADNI (Department of Defense Award No. W81XWH-12-2-0012).

\section{Competing interests}

The authors declare that they have no conflict of interest.

\section{Availability of data and materials}

The data used in the preparation of this article were obtained from the Alzheimer's disease Neuroimaging Initiative (ADNI) database: http://adni.loni.usc.edu/.

\section{References}

Anderkova, L., Barton, M., and Rektorova, I. (2017). Striato-cortical connections in Parkinson's and Alzheimer's diseases: Relation to cognition. Mov Disord 32(6), 917-922.

Balfour, D.J.K. (2004). The neurobiology of tobacco dependence: A preclinical perspective on the role of the dopamine projections to the nucleus. Nicotine \& Tobacco Research 6(6), 899-912.

Brody, A.L., Olmstead, R.E., London, E.D., Farahi, J., Meyer, J.H., Grossman, P., et al. (2004). Smoking-induced ventral striatum dopamine release. Am J Psychiatry 161(7), 1211-1218.

Buckner, R.L., Andrews-Hanna, J.R., and Schacter, D.L. (2008). The brain's default network: anatomy, function, and relevance to disease. Ann N Y Acad Sci 1124, 1-38.

Chhatwal, J.P., Schultz, A.P., Johnson, K.A., Hedden, T., Jaimes, S., Benzinger, T.L.S., et al. (2018). Preferential degradation of cognitive networks differentiates Alzheimer's disease from ageing. Brain 141(5), 1486- 
1500.

Claus, E.D., Blaine, S.K., Filbey, F.M., Mayer, A.R., and Hutchison, K.E. (2013). Association between nicotine dependence severity, BOLD response to smoking cues, and functional connectivity. Neuropsychopharmacology 38(12), 2363-2372.

Conti, A.A., McLean, L., Tolomeo, S., Steele, J.D., and Baldacchino, A. (2019). Chronic tobacco smoking and neuropsychological impairments: A systematic review and meta-analysis. Neurosci Biobehav Rev 96, 143-154.

Dennis, E.L., and Thompson, P.M. (2014). Functional brain connectivity using fMRI in aging and Alzheimer's disease. Neuropsychol Rev 24(1), 49-62.

Desmurget, M., and Sirigu, A. (2012). Conscious motor intention emerges in the inferior parietal lobule. Curr Opin Neurobio/ 22(6), 1004-1011.

Engelmann, J.M., Versace, F., Robinson, J.D., Minnix, J.A., Lam, C.Y., Cui, Y., et al. (2012). Neural substrates of smoking cue reactivity: a meta-analysis of fMRI studies. Neuroimage 60(1), 252-262.

Gallinat, J., Meisenzahl, E., Jacobsen, L.K., Kalus, P., Bierbrauer, J., Kienast, T., et al. (2006). Smoking and structural brain deficits: a volumetric MR investigation. Eur J Neurosci 24(6), 1744-1750.

Haber, S.N. (2016). Corticostriatal circuitry. Dialogues Clin Neurosci 18(1), 7-21.

Haber, S.N., and Knutson, B. (2010). The reward circuit: linking primate anatomy and human imaging. Neuropsychopharmacology 35(1), 4-26.

Hampel, H., Mesulam, M.M., Cuello, A.C., Farlow, M.R., Giacobini, E., Grossberg, G.T., et al. (2018). The cholinergic system in the pathophysiology and treatment of Alzheimer's disease. Brain 141(7), 1917-1933.

Hong, L.E., Gu, H., Yang, Y., Ross, T.J., Salmeron, B.J., Buchholz, B., et al. (2009). Association of nicotine addiction and nicotine's actions with separate cingulate cortex functional circuits. Arch Gen Psychiatry66(4), 431441. 
Ibrahim, B., Suppiah, S., Ibrahim, N., Mohamad, M., Hassan, H.A., Nasser, N.S., et al. (2021). Diagnostic power of resting-state $\mathrm{fMRI}$ for detection of network connectivity in Alzheimer's disease and mild cognitive impairment: A systematic review. Hum Brain Mapp 42(9), 2941-2968.

Jasinska, A.J., Stein, E.A., Kaiser, J., Naumer, M.J., and Yalachkov, Y. (2014). Factors modulating neural reactivity to drug cues in addiction: a survey of human neuroimaging studies. Neurosci Biobehav Rev 38, 1-16.

Knopman, D.S. (2019). Lowering of Amyloid-Beta by $\beta$-Secretase Inhibitors - Some Informative Failures. N Engl J Med 380(15), 1476-1478.

Larrieu, S., Letenneur, L., Orgogozo, J.M., Fabrigoule, C., Amieva, H., Le Carret, N., et al. (2002). Incidence and outcome of mild cognitive impairment in a population-based prospective cohort. Neurology 59(10), 1594-1599.

Liao, W., Wu, G.R., Xu, Q., Ji, G.J., Zhang, Z., Zang, Y.F., et al. (2014). DynamicBC: a MATLAB toolbox for dynamic brain connectome analysis. Brain Connect 4(10), 780-790.

Livingstone, P.D., and Wonnacott, S. (2009). Nicotinic acetylcholine receptors and the ascending dopamine pathways. Biochem Pharmaco/78(7), 744-755.

Looi, J.C., and Walterfang, M. (2013). Striatal morphology as a biomarker in neurodegenerative disease. Mol Psychiatry 18(4), 417-424.

McDonough, I.M., Wood, M.M., and Miller, W.S., Jr. (2019). A Review on the Trajectory of Attentional Mechanisms in Aging and the Alzheimer's Disease Continuum through the Attention Network Test. Yale J Biol Med 92(1), 37-51.

Mehta, M., Adem, A., Kahlon, M.S., and Sabbagh, M.N. (2012). The nicotinic acetylcholine receptor: smoking and Alzheimer's disease revisited. Front Biosci (Elite Ed) 4, 169-180.

Nadar, M.S., Hasan, A.M., and Alsaleh, M. (2021). The negative impact of chronic tobacco smoking on adult neuropsychological function: a cross-sectional study. BMC Public Health 21(1), 1278. 
Nelson, A.P., and O'Connor, M.G. (2008). Mild cognitive impairment: a neuropsychological perspective. CNS Spectr 13(1), 56-64.

Ninomiya, T., Sawamura, H., Inoue, K., and Takada, M. (2012). Segregated pathways carrying frontally derived top-down signals to visual areas MT and V4 in macaques. J Neurosci 32(20), 6851-6858.

Okita, K., Mandelkern, M.A., and London, E.D. (2016). Cigarette Use and Striatal Dopamine D2/3 Receptors: Possible Role in the Link between Smoking and Nicotine Dependence. Int J Neuropsychopharmacol 19(11).

Palmqvist, S., Schöll, M., Strandberg, O., Mattsson, N., Stomrud, E., Zetterberg, H., et al. (2017). Earliest accumulation of $\beta$-amyloid occurs within the default-mode network and concurrently affects brain connectivity. Nat Commun 8(1), 1214.

Pan, P., Zhu, L., Yu, T., Shi, H., Zhang, B., Qin, R., et al. (2017). Aberrant spontaneous low-frequency brain activity in amnestic mild cognitive impairment: A meta-analysis of resting-state fMRI studies. Ageing Res Rev $35,12-21$.

Pontieri, F.E., Tanda, G., Orzi, F., and Di Chiara, G. (1996). Effects of nicotine on the nucleus accumbens and similarity to those of addictive drugs. Nature 382(6588), 255-257.

Qiu, T., Luo, X., Shen, Z., Huang, P., Xu, X., Zhou, J., et al. (2016). Disrupted Brain Network in Progressive Mild Cognitive Impairment Measured by Eigenvector Centrality Mapping is Linked to Cognition and Cerebrospinal Fluid Biomarkers. J Alzheimers Dis 54(4), 1483-1493.

Quan, M., Zhao, T., Tang, Y., Luo, P., Wang, W., Qin, Q., et al. (2020). Effects of gene mutation and disease progression on representative neural circuits in familial Alzheimer's disease. Alzheimers Res Ther12(1), 14.

Rusanen, M., Kivipelto, M., Quesenberry, C.P., Jr., Zhou, J., and Whitmer, R.A. (2011). Heavy smoking in midlife and long-term risk of Alzheimer disease and vascular dementia. Arch Intern Med 171(4), 333-339. 
Steiner, H., and Van Waes, V. (2013). Addiction-related gene regulation: risks of exposure to cognitive enhancers vs. other psychostimulants. Prog Neurobio/ 100, 60-80.

Subramaniyan, M., and Dani, J.A. (2015). Dopaminergic and cholinergic learning mechanisms in nicotine addiction. Ann N Y Acad Sci 1349, 46-63.

Sullivan, M.D., Anderson, J.A.E., Turner, G.R., and Spreng, R.N. (2019). Intrinsic neurocognitive network connectivity differences between normal aging and mild cognitive impairment are associated with cognitive status and age. Neurobiol Aging 73, 219-228.

Tam, A., Dansereau, C., Badhwar, A., Orban, P., Belleville, S., Chertkow, H., et al. (2015). Common Effects of Amnestic Mild Cognitive Impairment on Resting-State Connectivity Across Four Independent Studies. Front Aging Neurosci 7, 242.

Wang, D., Belden, A., Hanser, S.B., Geddes, M.R., and Loui, P. (2020). Resting-State Connectivity of Auditory and Reward Systems in Alzheimer's Disease and Mild Cognitive Impairment. Front Hum Neurosci 14, 280.

Wang, P., Zhou, B., Yao, H., Zhan, Y., Zhang, Z., Cui, Y., et al. (2015). Aberrant intra- and inter-network connectivity architectures in Alzheimer's disease and mild cognitive impairment. Sci Rep 5, 14824.

Yalachkov, Y., Kaiser, J., Görres, A., Seehaus, A., and Naumer, M.J. (2013). Sensory modality of smoking cues modulates neural cue reactivity. Psychopharmacology (Berl) 225(2), 461-471.

Yalachkov, Y., Kaiser, J., and Naumer, M.J. (2009). Brain regions related to tool use and action knowledge reflect nicotine dependence. J Neurosci 29(15), 4922-4929.

Yan, C.G., Wang, X.D., Zuo, X.N., and Zang, Y.F. (2016). DPABI: Data Processing \& Analysis for (Resting-State) Brain Imaging. Neuroinformatics 14(3), 339-351.

Zhang, T., Luo, X., Zeng, Q., Fu, Y., Li, Z., Li, K., et al. (2020). Effects of Smoking on Regional Homogeneity in Mild Cognitive Impairment: A Resting-State Functional MRI Study. Front Aging Neurosci 12, 572732.

Zhen, D., Xia, W., Yi, Z.Q., Zhao, P.W., Zhong, J.G., Shi, H.C., et al. (2018). Alterations of brain local functional 
connectivity in amnestic mild cognitive impairment. Trans/ Neurodegener 7, 26.

Zhou, J., and Seeley, W.W. (2014). Network dysfunction in Alzheimer's disease and frontotemporal dementia: implications for psychiatry. Biol Psychiatry 75(7), 565-573.

Zhu, D.C., Majumdar, S., Korolev, I.O., Berger, K.L., and Bozoki, A.C. (2013). Alzheimer's disease and amnestic mild cognitive impairment weaken connections within the default-mode network: a multi-modal imaging study. J Alzheimers Dis 34(4), 969-984. 
Fig. 1. The interaction effects of smoking status $\times$ disease on $\mathrm{rsFC}$ of the striatal subregions. (A) between right caudate and left IPL; (B) between right putamen and bilateral cuneus; (C) between left NAc and bilateral ACC; and (D) between right NAc and bilateral ACC. IPL, inferior parietal lobule; NAc, nucleus accumbens; ACC, anterior cingulate cortex. The statistical threshold was set at $p<0.005$ with a cluster-level of $p<0.05$ (two-tailed, GRF corrected).

Fig. 2. The post-hoc analysis of rsFC values of the interaction regions. The $\mathrm{CN}$ smokers showed increased rsFC of right caudate to left IPL than other subgroups (A). The MCI smokers showed decreased rsFC of right putamen to cuneus (B) and increased rsFC of bilateral NAc to ACC (C and D) compared to other subgroups. IPL, inferior parietal lobule; NAc, nucleus accumbens; ACC, anterior cingulate cortex. ${ }^{*} p<0.05,{ }^{* *} p<0.01,{ }^{* * *} p<0.005$.

Fig. 3. Correlation between $\mathrm{rsFC}$ of the striatal subregions and neuropsychological scores. In MCI smokers, the rsFC value between left NAc and ACC was positively correlated with language ( $\mathrm{SVF}, \mathrm{r}=0.387, p=0.026)$; the $\mathrm{rsFC}$ value between right NAc and ACC was positively associated with language $(\mathrm{SVF}, \mathrm{r}=0.390, p=0.025)$ and memory (WMS-LM immediate recall, $\mathrm{r}=0.378, p=0.03$; delayed recall, $\mathrm{r}=0.367, p=0.036$ ). NAc, nucleus accumbens; ACC, anterior cingulate cortex; WMS-LM, Wechsler memory scale-logical memory; SVF, Semantic Verbal Fluency. 
Table 1. The demographic and clinical characteristics.

\begin{tabular}{|c|c|c|c|c|c|c|}
\hline Variables & $\begin{array}{c}\mathrm{CN} \\
\text { non-smokers } \\
(\mathrm{n}=304) \\
\end{array}$ & $\begin{array}{c}\mathrm{CN} \\
\text { smokers } \\
(\mathrm{n}=44) \\
\end{array}$ & $\begin{array}{c}\mathrm{MCI} \\
\text { non-smokers } \\
(\mathrm{n}=130)\end{array}$ & $\begin{array}{c}\text { MCI } \\
\text { smokers } \\
(\mathrm{n}=33) \\
\end{array}$ & $\mathrm{F} / \chi^{2}$ & $p$ \\
\hline \multicolumn{7}{|l|}{ Demographic factors } \\
\hline Age(years) & $72.98 \pm 7.25$ & $75.83 \pm 7.64$ & $74.33 \pm 7.87$ & $76.61 \pm 7.54$ & 4.09 & $0.007^{\mathrm{b}}$ \\
\hline $\operatorname{Sex}(\mathrm{F}: M)$ & $183: 121$ & $21: 23$ & $56: 74$ & $12: 21$ & 15.62 & 0.001 \\
\hline Education(years) & $16.88 \pm 2.33$ & $16.27 \pm 2.61$ & $16.71 \pm 2.54$ & $15.79 \pm 2.40$ & 2.56 & 0.054 \\
\hline \multicolumn{7}{|l|}{ Vascular risk factors } \\
\hline Hypertension, n(\%) & $118(38.80)$ & $21(47.70)$ & $59(45.40)$ & $20(60.60)$ & 7.04 & 0.071 \\
\hline Diabetes mellitus, $\mathrm{n}(\%)$ & $7(2.30)$ & $1(2.30)$ & $5(3.80)$ & $0(0.00)$ & 1.84 & 0.607 \\
\hline Hypercholesterolemia, $\mathrm{n}(\%)$ & $149(49.00)$ & $22(50.00)$ & $73(56.20)$ & $20(60.60)$ & 3.01 & 0.390 \\
\hline \multicolumn{7}{|l|}{ Neuropsychological tests } \\
\hline \multicolumn{7}{|l|}{ Memory } \\
\hline WMS-LM immediate recall & $14.64 \pm 3.61$ & $15.23 \pm 3.36$ & $10.62 \pm 4.30$ & $11.64 \pm 4.76$ & 38.55 & $<0.001^{\mathrm{abcd}}$ \\
\hline WMS-LM delayed recall & $13.53 \pm 3.80$ & $14.20 \pm 3.62$ & $8.58 \pm 4.37$ & $9.61 \pm 4.59$ & 54.94 & $<0.001^{\mathrm{abcd}}$ \\
\hline \multicolumn{7}{|l|}{ Attention } \\
\hline TMT-A & $31.30 \pm 9.51$ & $29.84 \pm 6.69$ & $36.15 \pm 13.05$ & $37.76 \pm 12.91$ & 9.96 & $<0.001^{\mathrm{abcc}}$ \\
\hline \multicolumn{7}{|l|}{ Execution } \\
\hline TMT-B & $74.85 \pm 35.90$ & $70.50 \pm 27.23$ & $96.23 \pm 51.98$ & $110.39 \pm 64.58$ & 14.21 & $<0.001^{\mathrm{abcc}}$ \\
\hline \multicolumn{7}{|l|}{ Language } \\
\hline SVF (animal) & $21.68 \pm 5.12$ & $21.32 \pm 5.87$ & $19.02 \pm 5.07$ & $18.67 \pm 5.23$ & 10.08 & $<0.001^{\mathrm{ab}}$ \\
\hline Head Motion (FD value) & $0.11 \pm 0.07$ & $0.12 \pm 0.07$ & $0.11 \pm 0.06$ & $0.13 \pm 0.07$ & 1.04 & 0.374 \\
\hline
\end{tabular}

Values are expressed as mean \pm standard deviation, number or percentage of participants.

Abbreviation: CN, Cognitively normal; MCI, Mild Cognitive Impairment; WMS-LM, Wechsler memory scale-logical memory; TMT, Trail-Making Test; SVF, Semantic Verbal Fluency; FD, framewise displacement.

${ }^{\mathrm{a}-\mathrm{d} P o s t ~ h o c ~ a n a l y s i s ~ f u r t h e r ~ r e v e a l e d ~ t h e ~ s o u r c e ~ o f ~ A N O V A ~ d i f f e r e n c e ~}\left({ }^{\mathrm{a}} \mathrm{CN}\right.$ non-smokers vs. MCI non-smokers; ${ }^{\mathrm{b}} \mathrm{CN}$ non-smokers vs. MCI smokers; ${ }^{\mathrm{c}} \mathrm{CN}$ smokers vs. MCI non-smokers; ${ }^{\mathrm{d}} \mathrm{CN}$ smokers vs. MCI smokers) $(p<0.05$, significant difference between the two groups) 
Table 2. Smoking status $\times$ disease interaction on $\mathrm{rsFC}$ of the striatal subregions.

\begin{tabular}{llccccc}
\hline \multirow{2}{*}{ Seeds } & Interaction effect regions & \multicolumn{3}{c}{ Peak MNI coordinate } & \multirow{2}{*}{ Peak intensity } & \multirow{2}{*}{ Cluster voxels } \\
\cline { 3 - 5 } & & $\mathrm{X}$ & $\mathrm{Y}$ & $\mathrm{Z}$ & & \\
\hline Right caudate & Left IPL & -42 & -54 & 54 & 18.5931 & 21 \\
Right putamen & Bilateral cuneus & 6 & -81 & 27 & 14.368 & 42 \\
Left NAc & Bilateral ACC & -3 & 21 & 24 & 15.4675 & 35 \\
Right NAc & Bilateral ACC & -3 & 18 & 27 & 20.4041 & 28 \\
\hline
\end{tabular}

The statistical threshold was set at $p<0.005$ with a cluster-level of $p<0.05$ (two-tailed, GRF corrected). MNI, Montreal Neurological Institute; IPL, inferior parietal lobule; ACC, anterior cingulate cortex. 
Table 3. Correlations between rsFC values and neuropsychological scores.

\begin{tabular}{lccccc}
\hline & $\begin{array}{c}\text { WMS-LM } \\
\text { immediate recall }\end{array}$ & $\begin{array}{c}\text { WMS-LM } \\
\text { delayed recall }\end{array}$ & TMT-A & TMT-B & SVF \\
\hline Across all groups & -0.059 & -0.025 & 0.099 & 0.035 & -0.042 \\
Left IPL & -0.028 & -0.019 & 0.054 & 0.034 & -0.045 \\
Bilateral cuneus & -0.024 & -0.03 & 0.098 & 0.031 & 0.042 \\
Bilateral ACC & -0.003 & -0.007 & 0.063 & 0.01 & 0.003 \\
Bilateral ACC & & & & \\
CN smokers & 0.178 & 0.115 & 0.241 & 0.147 & 0.096 \\
Left IPL & -0.229 & -0.221 & -0.034 & 0.156 & 0.14 \\
Bilateral cuneus & 0.203 & 0.169 & -0.298 & -0.177 & 0.131 \\
Bilateral ACC & 0.021 & 0.062 & -0.144 & -0.029 & -0.129 \\
Bilateral ACC & & & & & \\
MCI smokers & -0.027 & 0.009 & 0.078 & 0.27 & -0.096 \\
Left IPL & 0.174 & 0.167 & -0.199 & 0.11 & -0.026 \\
Bilateral cuneus & 0.145 & 0.153 & 0.046 & -0.257 & $\mathbf{0 . 3 8}^{*}$ \\
Bilateral ACC & a. & $\mathbf{0 . 3 6 7 ^ { * }}$ & 0.03 & -0.261 & $\mathbf{0 . 3 9}^{*}$ \\
Bilateral ACC $^{\mathrm{b}}$ & $\mathbf{0 . 3 7 8}^{*}$ & & & & \\
\hline
\end{tabular}

Data represent correlation coefficients. WMS-LM, Wechsler memory scale-logical memory; TMT, Trail-Making Test; SVF, Semantic Verbal Fluency; IPL, inferior parietal lobule; ACC, anterior cingulate cortex. ${ }^{a}$ represents rsFC value between left NAc and bilateral ACC. ${ }^{b}$ represents rsFC value between right NAc and bilateral ACC. ${ }^{*} p<0.05$, uncorrected. 

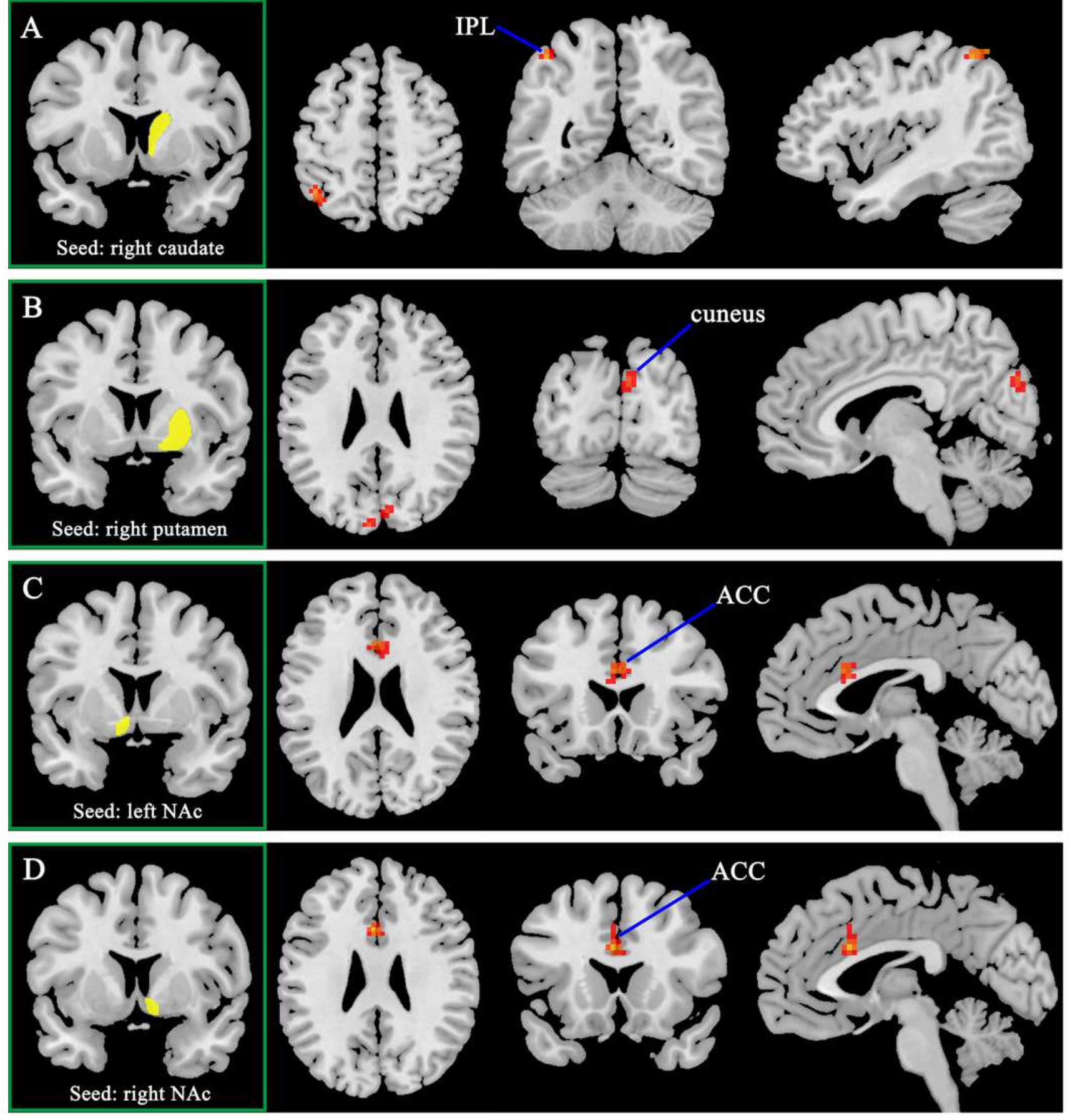

Figure 1

The interaction effects of smoking status $x$ disease on rsFC of the striatal subregions. (A) between right caudate and left IPL; (B) between right putamen and bilateral cuneus; $(C)$ between left NAc and bilateral $A C C$; and (D) between right NAC and bilateral ACC. IPL, inferior parietal lobule; NAc, nucleus accumbens; ACC, anterior cingulate cortex. The statistical threshold was set at $p<0.005$ with a cluster-level of $p<$ 0.05 (two-tailed, GRF corrected). 
A

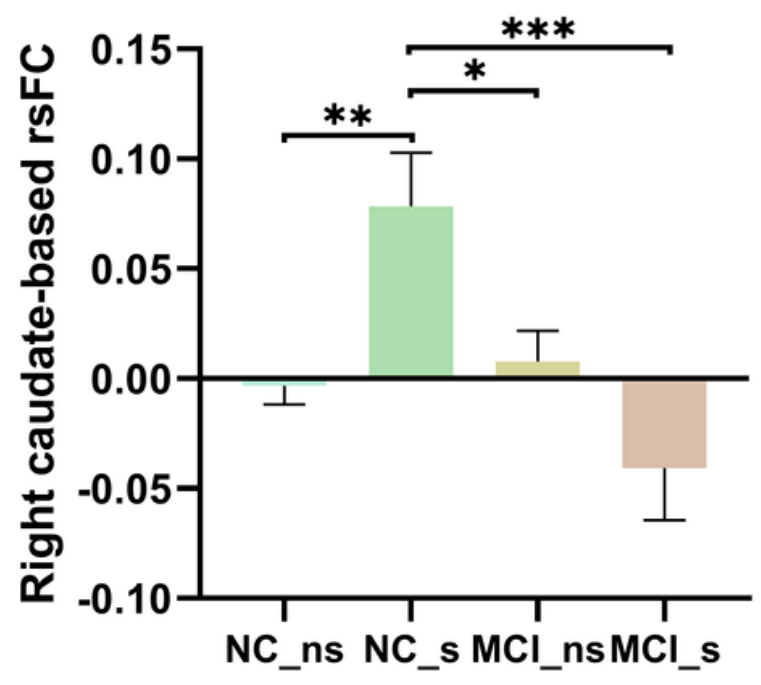

C

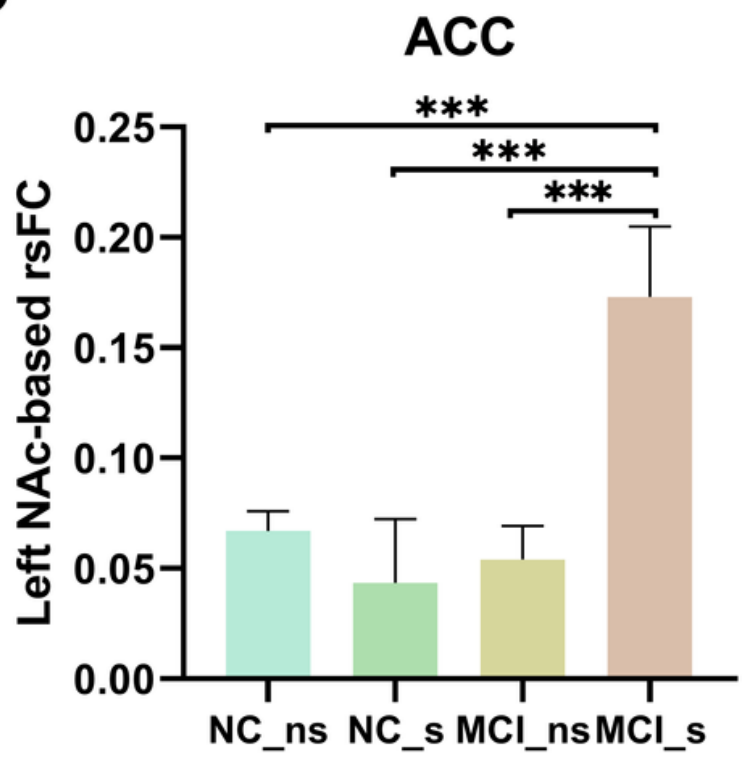

B

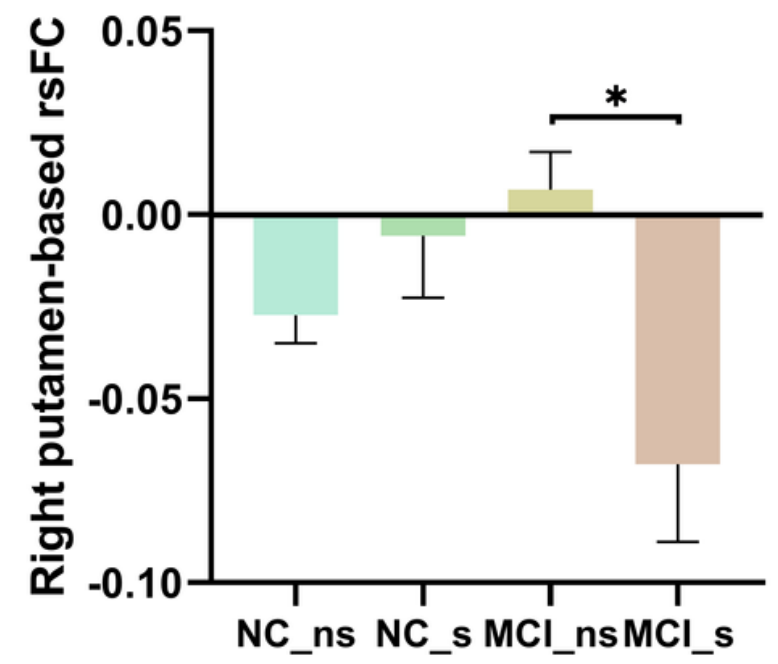

D

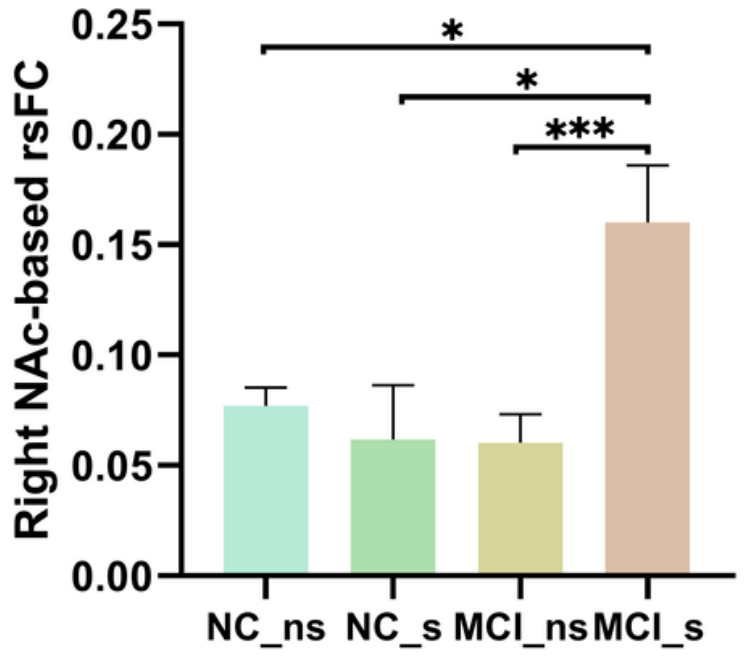

Figure 2

The post-hoc analysis of rsFC values of the interaction regions. The $\mathrm{CN}$ smokers showed increased rsFC of right caudate to left IPL than other subgroups (A). The $\mathrm{MCl}$ smokers showed decreased rsFC of right putamen to cuneus (B) and increased rsFC of bilateral NAc to ACC (C and D) compared to other subgroups. IPL, inferior parietal lobule; NAc, nucleus accumbens; ACC, anterior cingulate cortex. ${ }^{*} \mathrm{p}<0.05$, $\star * p<0.01, * \star * p<0.005$. 

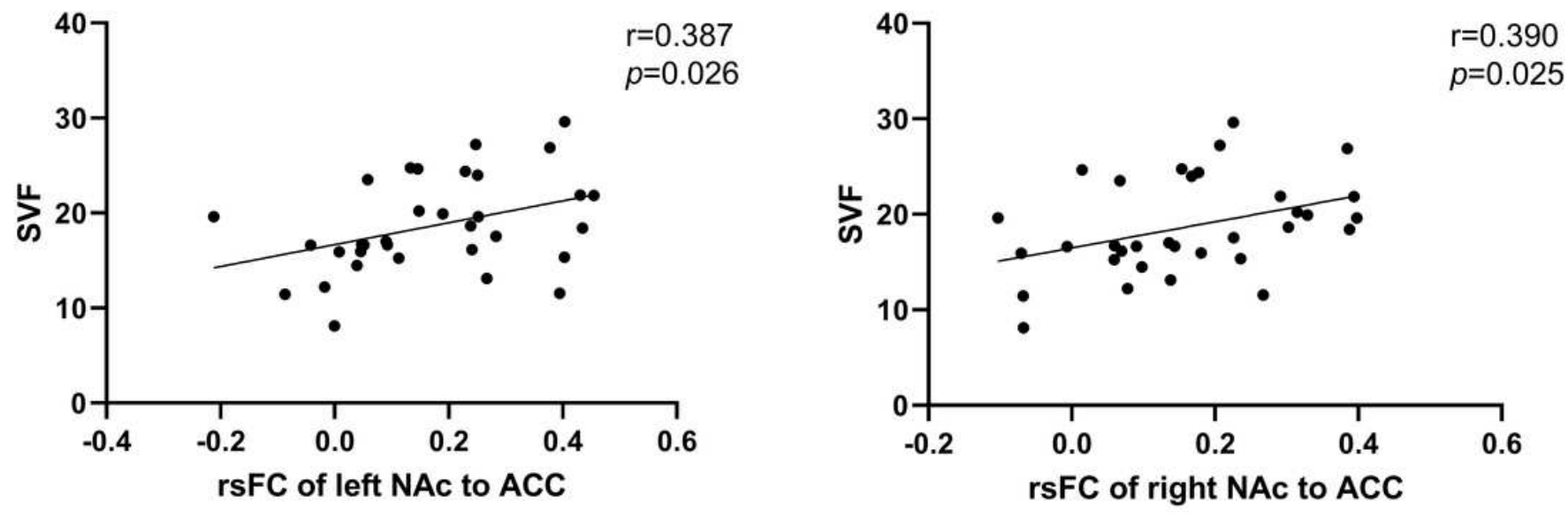

C

D
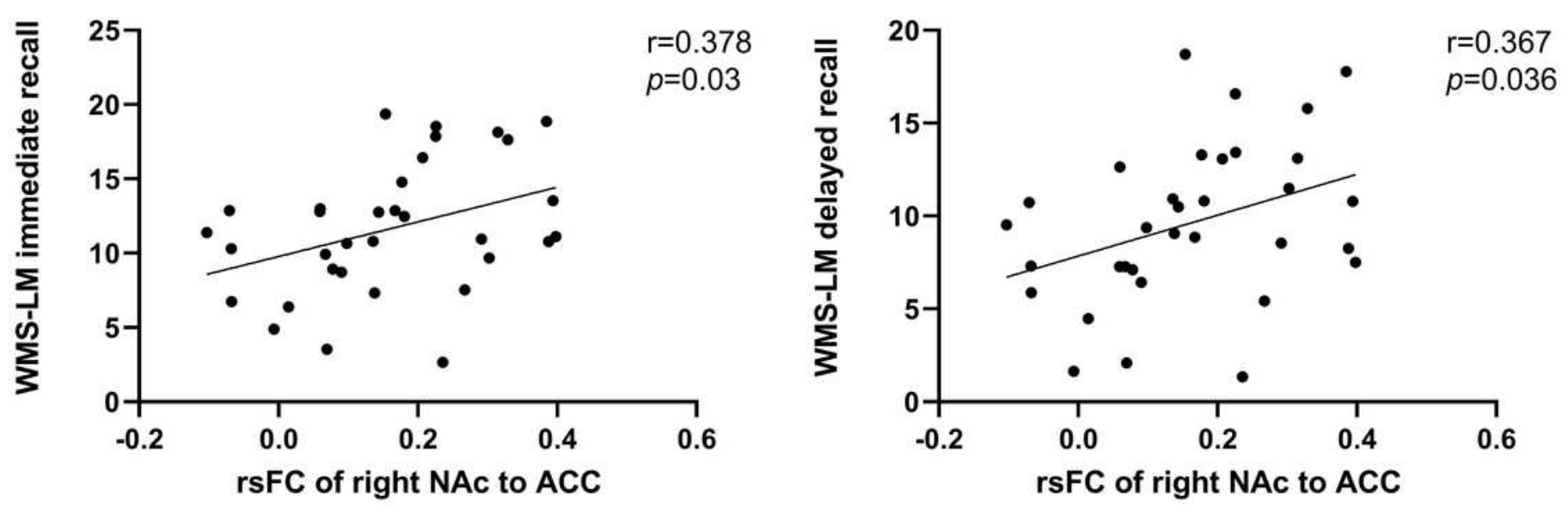

Figure 3

Correlation between rsFC of the striatal subregions and neuropsychological scores. In $\mathrm{MCl}$ smokers, the rsFC value between left NAc and ACC was positively correlated with language (SVF, $r=0.387, p=0.026)$; the rsFC value between right NAc and ACC was positively associated with language (SVF, $r=0.390, p=$ 0.025 ) and memory (WMS-LM immediate recall, $r=0.378, p=0.03$; delayed recall, $r=0.367, p=0.036$ ). NAc, nucleus accumbens; ACC, anterior cingulate cortex; WMS-LM, Wechsler memory scale-logical memory; SVF, Semantic Verbal Fluency.

\section{Supplementary Files}

This is a list of supplementary files associated with this preprint. Click to download.

- AuthorChecklist.pdf

- supplementarymaterials.pdf 